\title{
Which Fixation Device is Preferred for Surgical Treatment of Intertrochanteric Hip Fractures in the United States? A Survey of Orthopaedic Surgeons
}

\author{
Emily Niu MD, Arthur Yang MS, Alex H. S. Harris PhD, Julius Bishop MD
}

Received: 21 February 2015/Accepted: 15 July 2015/Published online: 25 July 2015

(C) The Association of Bone and Joint Surgeons $\mathbb{R} 2015$

\begin{abstract}
Background The best treatment for intertrochanteric hip fractures is controversial. The use of cephalomedullary nails has increased, whereas use of sliding hip screws has decreased despite the lack of evidence that cephalomedullary nails are more effective. As current orthopaedic trainees receive less exposure to sliding hip screws, this may continue to perpetuate the preferential use of cephalomedullary nails, with important implications for resident education, evidence-based best practices, and healthcare cost.

Questions/purposes We asked: (1) What are the current practice patterns in surgical treatment of intertrochanteric fractures among orthopaedic surgeons? (2) Do surgical practice patterns differ based on surgeon characteristics, practice setting, and other factors? (3) What is the rationale behind
\end{abstract}

Each author certifies that he or she, or a member of his or her immediate family, has no funding or commercial associations (eg, consultancies, stock ownership, equity interest, patent/licensing arrangements, etc) that might pose a conflict of interest in connection with the submitted article.

All ICMJE Conflict of Interest Forms for authors and Clinical Orthopaedics and Related Research ${ }^{\mathbb{R}}$ editors and board members are on file with the publication and can be viewed on request.

Each author certifies that his or her institution approved or waived approval for the human protocol for this investigation and that all investigations were conducted in conformity with ethical principles of research.

Electronic supplementary material The online version of this article (doi:10.1007/s11999-015-4469-5) contains supplementary material, which is available to authorized users.

E. Niu ( $\varangle)$, A. Yang, A. H. S. Harris, J. Bishop

Department of Orthopaedic Surgery, Stanford University, 450

Broadway Street, M/C 6342, Redwood City, CA 94063, USA

e-mail: eniu@stanford.edu these surgical practice patterns? (4) What postoperative approaches do surgeons use for intertrochanteric fractures? Methods A web-based survey containing 20 questions was distributed to active members of the American Academy of Orthopaedic Surgeons. Three thousand sevenhundred eighty-six of 10,321 invited surgeons participated in the survey (37\%), with a $97 \%$ completion rate (3687 of 3784 responded to all questions in the survey). The survey elicited information regarding surgeon demographics, preferred management strategies, and decision-making rationale for intertrochanteric fractures.

Results Surgeons use cephalomedullary nails most frequently for treatment of intertrochanteric hip fractures. Sixty-eight percent primarily use cephalomedullary nails, whereas only $19 \%$ primarily use sliding hip screws, and the remaining $13 \%$ use cephalomedullary nails and sliding hip screws with equal frequency. The cephalomedullary nail was the dominant approach regardless of experience level or practice setting. Surgeons who practiced in a nonacademic setting ( $71 \%$ versus 58\%; p < 0.001), did not supervise residents $(71 \%$ versus $61 \% ; \mathrm{p}<0.001)$, or treated more than five intertrochanteric fractures a month $(78 \%$ versus $67 \% ; \mathrm{p}<0.001$ ) were more likely to use primarily cephalomedullary nails. Of the surgeons who used only cephalomedullary nails, ease of surgical technique (58\%) was cited as the primary reason, whereas surgeons who used only sliding hip screws cite familiarity (44\%) and improved outcomes $(37 \%)$ as their primary reasons. Of those who use only short cephalomedullary nails, ease of technique $(59 \%)$ was most frequently cited. Postoperatively, $67 \%$ allow the patient to bear weight as tolerated. Nearly all respondents $(99.5 \%)$ use postoperative chemical thromboprophylaxis.

Conclusions Despite that either sliding hip screw or cephalomedullary nail fixation are associated with 
equivalent outcomes for most intertrochanteric femur fractures, the cephalomedullary nail has emerged as the preferred construct, with the majority of surgeons believing that a cephalomedullary nail is easier to use, associated with improved outcomes, or is biomechanically superior to a sliding hip screw. The difference between what is evidence-based and what is done in clinical practice may be attributed to several factors, including financial considerations, educational experience, or inability of our current outcomes measures to reflect the experiences of surgeons. The educators, researchers, and policymakers among us must work harder to better define the roles of sliding hip screws and cephalomedullary nails and ensure that the increasing population with hip fractures receives highquality and economically responsible care.

Level of Evidence Level V, therapeutic study.

\section{Introduction}

Geriatric hip fractures are an increasing epidemic in the United States as the population ages, with an anticipated incidence of 500,000 fractures per year by 2040 [17]. Intertrochanteric fractures comprise approximately $50 \%$ of these. Historically, a sliding compression screw has been the preferred implant [19, 28, 30, 34]; however, cephalomedullary nailing has become an increasingly popular fixation technique for these fractures since its introduction in the 1980s [2, 7, 12]. Cephalomedullary nailing has theoretical advantages of being less invasive and biomechanically superior, providing a buttress to limit fracture collapse [21]. Multiple clinical trials and meta-analyses have directly compared the two techniques for treatment of these fractures [3, 18-20, 27, 28, 30, 34]. Particular fracture patterns, such as those with reverse obliquity, subtrochanteric extension, fracture extension into the lateral wall, or greater trochanteric avulsion [23, 26, 33], have lower rates of failure and reoperation when treated with a cephalomedullary nail than a sliding hip screw. However, to our knowledge, no study has found one construct to be clearly superior in the treatment of commonly encountered conventional obliquity fracture patterns, and controversy over the optimal treatment strategy persists $[3,5,25,27$, $28,30,32]$.

There is difficulty reaching a consensus based on available evidence given the heterogeneity of fracture patterns and implant designs reported in the literature [3, 18, 27, 30, 34]. Despite this uncertainty, data collected between 1999 and 2006 indicated that cephalomedullary nailing was overtaking use of sliding hip screws as the preferred technique for fixation of these fractures, particularly among younger surgeons [2]. We therefore wished to investigate current practice patterns among orthopaedic surgeons in the treatment of intertrochanteric fractures and the rationale behind decision-making. In particular, we hoped to identify the impetus for changing practice patterns. As the aging population strains our available healthcare resources, it is becoming increasingly important to deliver evidence-based and cost-effective care.

Specifically, we asked: (1) What are the current practice patterns in surgical treatment of intertrochanteric fractures among orthopaedic surgeons? (2) Do surgical practice patterns differ based on surgeon characteristics, practice setting, and other factors? (3) What is the rationale behind these surgical practice patterns? (4) What postoperative approaches do surgeons use for intertrochanteric fractures?

\section{Materials and Methods}

We conducted a cross-sectional survey study of current practices and opinions of orthopaedic surgeons in the treatment of standard obliquity intertrochanteric fractures. We developed a survey using previous literature and expert opinion (level $\mathrm{V}$ evidence). The final questionnaire consisted of 20 questions that were divided into three broad categories: surgeon demographics and training background (years in practice and practice type, number of intertrochanteric fractures treated), operative considerations in the treatment of intertrochanteric fractures including the rationale for decision-making (preference of cephalomedullary nails versus sliding hip screws, rationale for choosing one implant instead of the other), and postoperative management of weightbearing precautions and use of chemical thromboprophylaxis (Appendix 1. Supplemental material is available with the online version of CORR ${ }^{\circledR}$.). The question regarding the number of years in practice of the respondent featured an open answer field. The remainder of the questions offered close-ended, multiple choice-type responses. Questions pertaining to rationale for decision making included "Other" with an open response comment field as one of the multiple choice answers.

The survey was distributed by email to current North American members of the American Academy of Orthopaedic Surgeons (AAOS). Resident, nonpracticing, and retired members were excluded, as were international members. We targeted a broad demographic because these fractures are treated by orthopaedic surgeons with diverse backgrounds and practice settings.

After the initial email, we sent two reminder emails each spaced 2 weeks apart. The total collection period spanned 3 months. We designed the survey to maximize response and completion rates. The purpose of the study was clearly stated, questions were designed to be relevant and easy to 
answer, the sequence of questions was logical, and a webbased survey engine allowed respondents to be directed to certain questions based on their answers to previous questions, optimizing efficiency. Respondents were not allowed to advance to the next page until all questions on a given page were answered with the goal of maintaining a high completion rate.

Responses were collected and reviewed by one reviewer (EN). The variables studied included surgeon training background including fellowship and current practice setting. Surgeons also were asked about their rationale for decision-making, specifically in deciding between use of sliding hip screws and cephalomedullary nails and use of short versus long cephalomedullary nails. No detailed description or classification of the fracture or injury radiographs were provided. Rather surgeons were given the opportunity to respond "depends on fracture pattern". Surgeons also were asked about their postoperative management regarding weightbearing restrictions and venous thromboembolism prophylaxis.

A total of 3786 of $10,321(37 \%)$ invited surgeons completed the survey, with a $97 \%$ completion rate (3687 of 3784). All survey respondents (3786) answered the questions pertaining to demographics (Table 1). The average number of years in practice was 17 (range, 6 months to 57 years). Ninety-four percent (3568) of the respondents were male and 6\% (218) were female. There were notable variations in training and practice patterns. Seventeen percent (644) worked in an academic practice, whereas 83\% (3142) worked in nonacademic settings. Thirty percent (1126) of respondents supervised residents and $70 \%$ (2660) did not. Ten percent (363) completed a trauma fellowship, 56\% (2131) completed a nontrauma fellowship, and 34\% (1292) did not complete a fellowship. Seventyfive percent (2854) treated between zero and five intertrochanteric fractures a month, 20\% (746) treated more than five intertrochanteric fractures a month, and 5\% (186) stated that they do not commonly treat intertrochanteric fractures. In comparison to the AAOS 2014 census [1], our respondents were representative of the general AAOS membership, with an average age of practicing orthopaedists of 54.76 years, $92 \%$ male, and $16 \%$ in an academic setting. Our survey respondents featured a larger proportion of trauma-specialized surgeons compared with the AAOS membership (10\% vs $5 \%$ ), as expected given the subject matter.

Categorical data were summarized using percentages. Trends in preferences and practice patterns were analyzed and response distributions determined. Odds ratios (OR) and $95 \%$ CIs were used to determine statistical differences between proportions, with a $\mathrm{p}$ value less than 0.05 used to define statistical significance. Surgeons were stratified based on experience (number of years in practice, $<2$ years
Table 1. Demographics of the survey respondents and the AAOS 2014 Census [1]

\begin{tabular}{|c|c|c|}
\hline Variable & $\begin{array}{l}\text { Intertrochanteric } \\
\text { fracture survey }\end{array}$ & $\begin{array}{l}\text { American } \\
\text { Academy } \\
\text { of Orthopaedic } \\
\text { Surgeons } \\
2014 \text { census }\end{array}$ \\
\hline Average years in practice & $\begin{array}{l}17 \text { years (range, } \\
6 \text { months-57 } \\
\text { years) }\end{array}$ & Not asked \\
\hline Average age & Not asked & $\begin{array}{c}54.8 \text { years (range, } \\
22-98 \text { years) }\end{array}$ \\
\hline \multicolumn{3}{|l|}{ Sex } \\
\hline Male & $94 \%$ & $92 \%$ \\
\hline Female & $6 \%$ & $8 \%$ \\
\hline \multicolumn{3}{|l|}{ Practice setting } \\
\hline Academic & $17 \%$ & $16 \%$ \\
\hline Nonacademic & $83 \%$ & $84 \%$ \\
\hline Supervise residents & & Not asked \\
\hline Yes & $30 \%$ & \\
\hline No & $70 \%$ & \\
\hline \multicolumn{3}{|l|}{ Specialization } \\
\hline Trauma & $10 \%$ & $5 \%$ \\
\hline Nontrauma & $56 \%$ & $81 \%$ \\
\hline None & $34 \%$ & $14 \%$ \\
\hline $\begin{array}{l}\text { Number of fractures } \\
\text { treated/month }\end{array}$ & & Not asked \\
\hline Did not commonly treat & $5 \%$ & \\
\hline $0-5$ & $75 \%$ & \\
\hline$>5$ & $20 \%$ & \\
\hline
\end{tabular}

[ $\mathrm{n}=337$ ]; 2 to 5 years [ $\mathrm{n}=464]$ ]; 6 to 10 years $[\mathrm{n}=578$ ]; 11 to 20 years $[\mathrm{n}=1055]$; 21 to 30 years $[\mathrm{n}=903]$; and $>30$ years [n $=437]$ ) (Fig. 1), to determine the proportions who use sliding hip screws and cephalomedullary nails in their training and current practice. The surgeons with fewer than 10 years in practice were stratified into more groups owing to their large numbers and to determine whether there were any practice differences seen in the most recent residency graduates. Logistic regression analysis was performed to determine whether there was a correlation between surgeon experience and use of sliding hip screws versus cephalomedullary nails in training and in practice.

\section{Results}

In training, the majority of surgeons (55\%; 2088 of 3786) were taught using primarily sliding hip screws, compared with $33 \%$ who trained using primarily cephalomedullary nails (1262 of 3786, OR, 2.46; 95\% CI, 2.24-2.70; p < 0.001)(Fig. 2A). Surgeons use cephalomedullary nails most frequently in their current practice for treatment of 


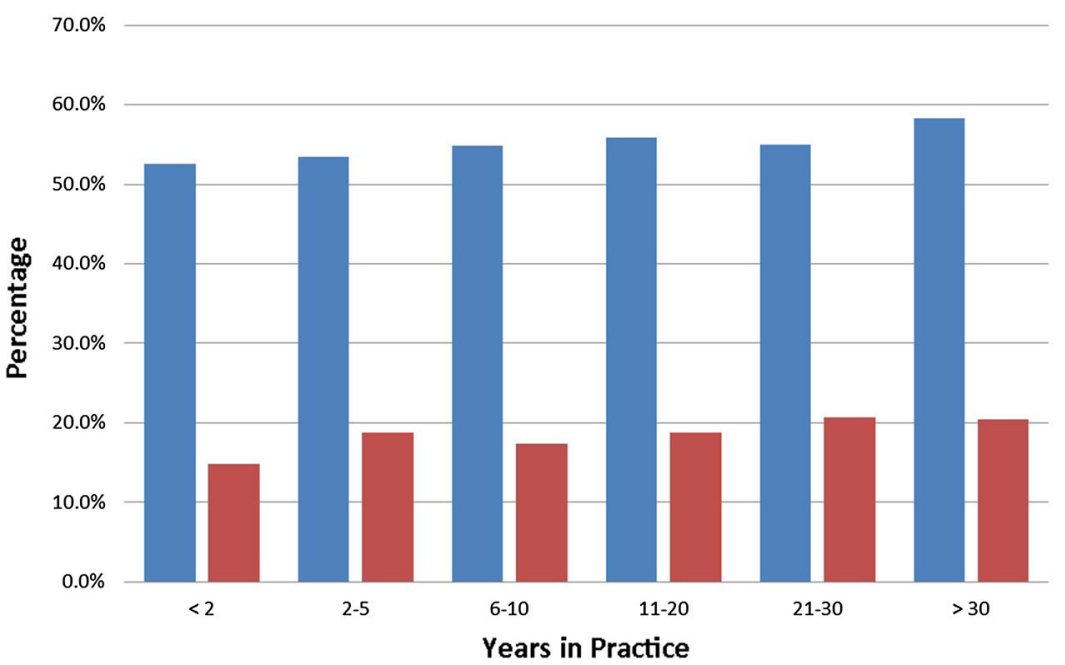

A

$\square$ Training $\square$ Current

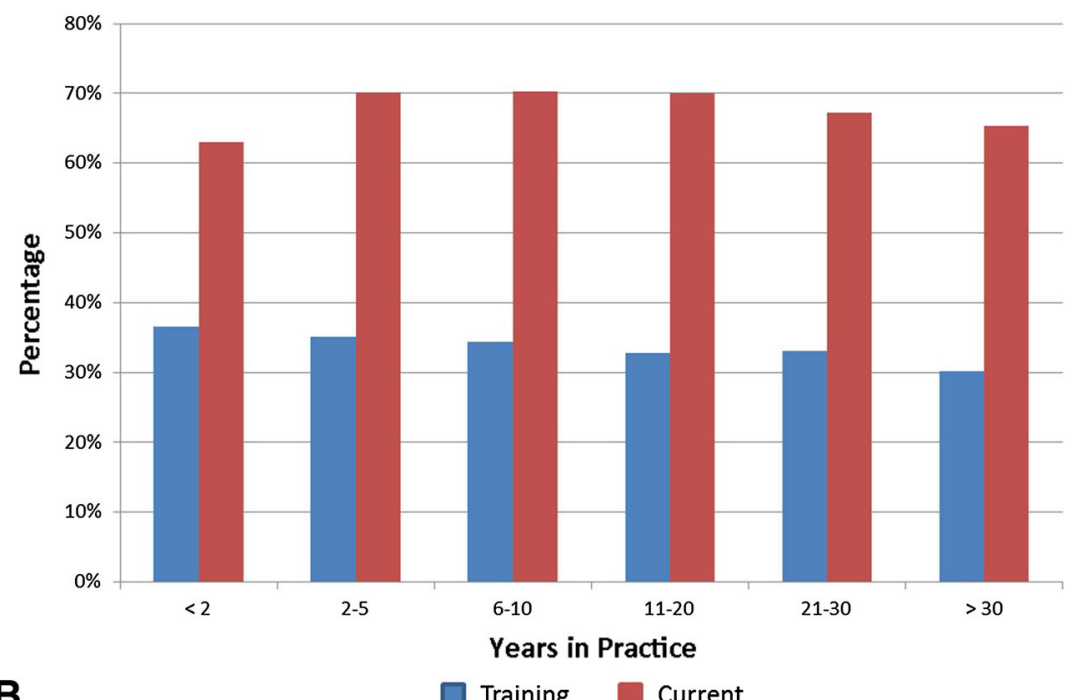

B

$\square$ Training $\square$ Current

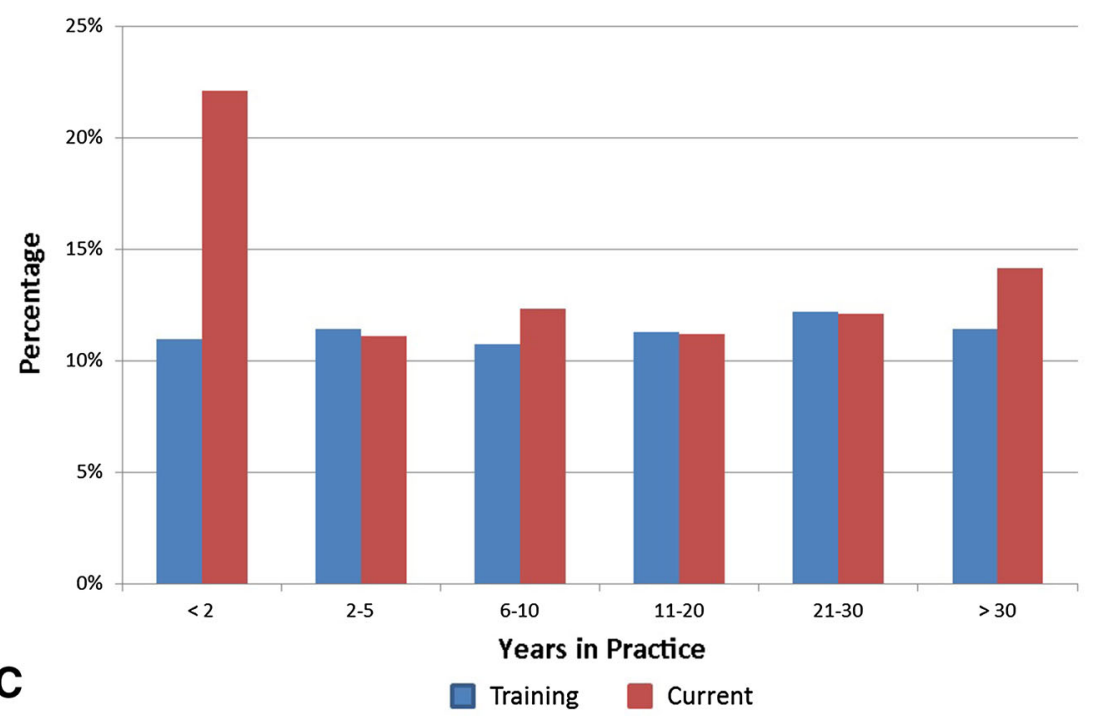


4Fig. 1A-C Rates of cephalomedullary nail (CMN) use were stratified across surgeon experience. Respondents were stratified by years in practice $(<2,2-5,6-10,11-20,21-30,>30)$. The proportion who use primarily sliding hip screws (SHS) (those who responded "SHS only" or "mostly SHS"), primarily cephalomedullary nails (responded "CMN only" or "mostly CMN"), and both (responded "I use SHS and CMN equally") were calculated for training and current practice in each experience group. The percentages of respondents who use primarily (A) sliding hip screws, (B) cephalomedullary nails, and (C) sliding hip screws and cephalomedullary nails equally in training versus in current practice are presented.

intertrochanteric hip fractures (Fig. 2B). Although 68\% (2533 of 3712) currently use primarily cephalomedullary nails, only $19 \%$ (698 of 3712) use primarily sliding hip screws to treat intertrochanteric fractures (OR, 9.28; 95\% CI, 8.33-10.33; $\mathrm{p}<0.001$ ). The remaining $13 \%$ (481 of 3712) use cephalomedullary nails and sliding hip screws equally in current practice (Fig. 2B). Among surgeons who use cephalomedullary nails, 29\% (1029 of 3548) use only long cephalomedullary nails, whereas 3\% (97 of 3548) use only short cephalomedullary nails, with the remaining $68 \%$ (2422 of 3548) using long and short cephalomedullary nails. Surgeons with more years in practice were more likely to have trained using sliding hip screws (Fig. 1A; OR of primarily using sliding hip screws in training with each increasing year in practice, $1.007 ; 95 \% \mathrm{CI}, 1.00$ to $1.01 ; \mathrm{p}=0.046$ ), however greater than $60 \%$ of surgeons currently use primarily cephalomedullary nails regardless of the number of years in practice (Fig. 1B). There was no correlation found between the number of years in practice and use of sliding hip screws versus cephalomedullary nails in current practice. Surgeons with less than 2 years in practice were more likely to use both constructs with equal frequency compared with other experience levels (Fig 1C) ( $<2$ years, $22 \%$ [73 of 330]; all other experience levels, $12 \%$ [404 of 3371]; OR, 2.09; 95\% CI, 1.58-2.76; p < 0.001 ).

We found that practice setting and training background affected surgeon use of cephalomedullary nails versus sliding hip screws. Surgeons who practiced in a nonacademic setting were more likely to use primarily cephalomedullary nails compared with those in an academic setting (72\% [2029 of 2814] versus 58\% [357 of 615], respectively; OR, 1.87; 95\% CI, 1.56-2.24; p < $0.001)$. Similarly surgeons who did not supervise residents (71\% [1859 of 2614] versus 61\% [674 of 1098]; OR, 1.55; 95\% CI, 1.34-1.80; $\mathrm{p}<0.001)$ or treated more than five intertrochanteric fractures a month (78\% [576 of 741] versus $66 \%$ [1957 of 2971]; OR, 1.81; 95\% CI, 1.50-2.18; $\mathrm{p}<0.001$ ) were more likely to use primarily cephalomedullary nails. Compared with other surgeons, surgeons who completed a trauma fellowship (16\% [57 of 360] versus $35 \%$ [1176 of 3352]; OR, 0.35 ; 95\% CI, 0.26-0.47; p < 0.001 ), practiced in an academic setting (13\% [80 of 615] versus $38 \%$ [1076 of 2814]; OR, 0.24; 95\% CI, 0.19-0.31; $\mathrm{p}<0.001)$, or supervised residents (19\% [204 of 1098] versus $39 \%$ [1029 of 2614]; OR, 0.35; 95\% CI, 0.35-0.42; $\mathrm{p}<0.001$ ) were less likely to use a single construct (only cephalomedullary nails or only sliding hip screws) exclusively. Surgeons were more likely to use only long cephalomedullary nails if they completed a trauma fellowship (42\% [149 of 359] versus 27\% [880 of 3252]; OR, $1.91 ; 95 \% \mathrm{CI}, 1.52-2.39 ; \mathrm{p}<0.001)$, practiced in an academic setting (41\% [244 of 601] versus $26 \%$ [701 of 2739]; OR, 1.99; 95\% CI, 1.65-2.39; $\mathrm{p}<0.001)$, or supervised residents (37\% [396 of 1079] versus 25\% [633 of 2532]; OR, 1.74; 95\% CI, 1.49-2.03; $\mathrm{p}<0.001)$.

The polled surgeons who favor cephalomedullary nails perceive them to be superior to sliding hip screws, whereas those who favor sliding hip screws did so because of their familiarity with the construct. Of the surgeons who use cephalomedullary nails exclusively (1106), the ease of surgical technique (58\%; 646 of 1106), improved outcomes compared with sliding hip screws (16\%; 178 of 1106), biomechanical superiority (15\%; 163 of 1106), and familiarity with technique (10\%; 106 of 1106) were identified as the primary reasons. Patient comorbidities $(1 \% ; 13$ of $1106)$ and perceived cost ( 0 of 1106) were not important influences on decision-making. Surgeons who use only sliding hip screws (127) chose familiarity with technique (44\%; 56 of 127), perceived improved or equivalent outcomes to cephalomedullary nails (38\%; 48 of 127), and surgical ease $(16 \% ; 20$ of 127$)$ as their reasons for favoring the sliding hip screws. Perceived cost (1\%; two of 127) and patient comorbidities (1\%; one of 127), were not primary factors in decision making. Of respondents who use only long cephalomedullary nails (1029), decreased risk of periimplant fracture (75\%; 767 of 1029), biomechanical superiority $(18 \% ; 181$ of 1029), and patient comorbidities (7\%; 72 of 1029) served as the rationale. Five respondents chose "familiarity with technique", and four chose "other", with responses including: "all of the above", "peer pressure", "dogma", and "I would use a DHS (dynamic hip screw) instead of a short nail because it is cheaper". Of respondents who use only short cephalomedullary nails (97), ease of technique (59\%; 57 of 97), and perceived equivalency of outcomes to a long nail $(31 \% ; 30$ of 97) drove decision-making. Decreased risk of intraoperative fracture (5\%; five of 97), cost (3\%; three of 97), and patient comorbidities (2\%; two of 97) were less-important factors.

Postoperatively, 67\% (2457 of 3687) allow the patient to bear weight as tolerated, $29 \%$ (1060 of 3687) allow toetouch or partial weightbearing, and $2 \%$ (60 of 3687) do not allow weightbearing. The remaining 3\% (110 of 3687) determine weightbearing precautions based on fracture pattern, with the majority selecting partial weightbearing 
Use of CMN vs SHS in Training

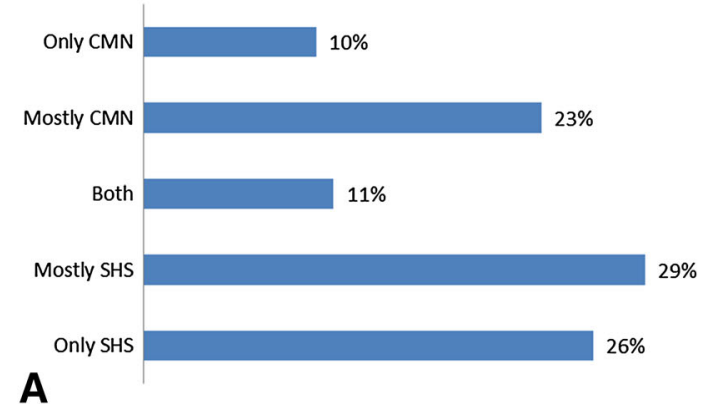

Fig. 2A-B Use of cephalomedullary nails (CMN) and sliding hip screws (SHS) in training and current practice are shown. The graphs show responses to the most common implant used (A) for treatment of intertrochanteric fractures by the surgeon while in training, and (B)

for more comminuted or unstable fracture patterns and weightbearing as tolerated for stable patterns. Surgeons who use primarily cephalomedullary nails were more likely to allow full weightbearing immediately after surgery $(70 \% ; 1759$ of 2522$)$ than those who use sliding hip screws (55\%; 381 of 689 ; OR, 1.86; 95\% CI, 1.57-2; p < 0.001). For venous thromboembolism prophylaxis, only $0.5 \%$ (17 of 3687) of those surveyed use mechanical prophylaxis alone. Fifty-five percent (2024 of 3687) use enoxaparin, $16 \%$ (596 of 3687) use warfarin, $10 \%$ (375 of 3687) use rivaroxaban, and $9 \%$ (317 of 3687) use aspirin. Ten percent (358 of 3687) use another form of chemical thromboprophylaxis. Fifty-five percent (2035 of 3674) use chemical thromboprophylaxis for 2 to 4 weeks, 26\% (958 of 3674) 1 to 2 weeks, $14 \%$ (528 of 3674) greater than 4 weeks, and $4 \%$ (153 of 3674) use it for less than 1 week.

\section{Discussion}

Despite extensive research and debate devoted to the subject $[3,5,18-20,25,27,28,30,32,34]$, neither cephalomedullary nails nor sliding hip screws have been established as the definitively superior construct for the treatment of intertrochanteric hip fractures. However, cephalomedullary nails are the most commonly used construct in the current treatment of intertrochanteric hip fractures, despite the lack of evidence and increased cost [32]. We conducted a survey to identify sources for the discrepancy between what is supported in the literature and what is used in practice. These findings may reflect factors that have not been widely recognized or studied, but that are important to surgeons, such as ease of surgical technique. We found that the cephalomedullary nail is the favored implant by surgeons across all experience levels. Surgeons in a nonacademic setting, who do not work with residents, and treat a higher volume of hip fractures are

\section{Use of CMN vs SHS in Current Practice}

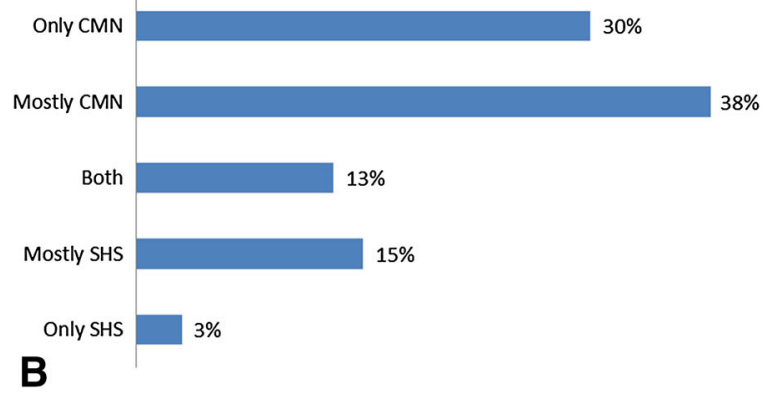

by the surgeon in current practice. There is a notable shift toward the use of cephalomedullary nails in current practice. Both = SHS and $\mathrm{CMN}$ are used with equal frequency.

even more likely to use cephalomedullary nails. Ease of surgical technique was the most frequently cited reason for use of cephalomedullary nails.

Our study has some limitations. The low response rate, although consistent with other surgical surveys $[4,14,20]$, could introduce responder bias. Our data may not be generalizable to the entire practicing population in the country; however, the survey does reflect the current treatment practices and perceptions of nearly 4000 surgeons. The demographics of our respondents also closely match the demographics of the current AAOS membership, based on 2014 census data [1], suggesting that we succeeded in achieving a broad and representative sampling of currently practicing orthopaedic surgeons. In addition, not all of the surgeons who were sampled are likely to treat hip fractures, which further offsets our responder bias limitation. Our survey did not detail particular fracture patterns or provide radiographs when querying surgeons about implant choice. Although a previous study polling surgeons showed there is no standard classification system that is used to define stable versus unstable fractures and no widely accepted treatment algorithm [20], we recognize that fracture pattern is an important consideration to many surgeons when they are selecting an implant and that the cephalomedullary nail can be used for certain fracture patterns (reverse obliquity, lateral wall involvement, subtrochanteric extension) where a sliding hip screw cannot be used. We addressed this by including the answer choice "depends on fracture pattern" in our questions regarding the rationale for treatment strategies. This answer option was not chosen frequently, which suggests that other factors are more important in guiding treatment. Similarly, we did not specifically ask surgeons about their practices with a geriatric patient population, but given that the majority of patients with intertrochanteric fractures are elderly, we think it is safe to apply data generated by our survey to this population. The survey design is reliant on self-reported data. We attempted 
to control for this by using close-ended, multiple choicetype questions to allow for comparability. However, this type of question limits the ability of respondents to report responses that did not appear on the survey. We offered comment fields after the majority of questions to address this limitation but these were used infrequently and did not significantly affect the results of our study. Additionally, we primarily used single-selection questions to elicit only the most relevant option for each scenario. Finally, because of the large number of responses, small but statistically significant differences may not always be clinically relevant. However, our reported comparison statistics all have a difference of $10 \%$ or greater.

Our finding that the majority of surgeons currently use cephalomedullary nails is in agreement with the literature showing that the popularity of the cephalomedullary nails is steadily increasing [2, 7, 12]. Anglen and Weinstein [2] conducted a study of candidates participating in the American Board of Orthopaedics oral boards and found the use of cephalomedullary nails increased from $3 \%$ to $65 \%$ from 1999 to 2006 by newly practicing orthopaedic surgeons. A study of treatment of intertrochanteric fractures in Medicare beneficiaries showed that the use of cephalomedullary nails more than doubled between 2000 and 2002 [12]. We found that surgeons across all experience levels preferentially use cephalomedullary nails in their current practice, suggesting that this trend is not only the result of an influx of younger surgeons using primarily cephalomedullary nails. Rather, even more experienced surgeons are favoring cephalomedullary nails. This reflects an overall shift in practice paradigm-one that does not appear to be supported by the current literature.

We found that surgeons at academic centers or who supervise residents were more likely to use both constructs. Our findings are in contrast to those of Forte et al. [11] who found, in a study conducted between 2000 and 2002 on Medicare beneficiaries, that teaching hospital status and resident assistance during surgery are associated with increased cephalomedullary nail use. It remains to be seen whether future generations of surgeons will continue the trend in preferentially using cephalomedullary nails despite exposure to both techniques, or whether lack of literature support for indiscriminant use of cephalomedullary nails will create enough momentum to swing the pendulum back toward use of sliding hip screws.

Our data show that surgeons believe that cephalomedullary nails are a superior construct to sliding hip screws, whether attributable to ease of technique, perceived improved outcomes, or biomechanical advantage. There continues to be controversy regarding the optimum treatment of intertrochanteric fractures [3, 5, 25, 27, 28, 30, 32]. Several large prospective randomized trials have directly compared sliding hip screws and contemporary cephalomedullary nail constructs and have shown similar outcomes between the two techniques [3, 27, 28, 30, 34]. The most recent Cochrane review on the topic [28] concluded that the sliding hip screw is the superior construct for treatment of intertrochanteric fractures because of the decreased rate of surgical complications compared with cephalomedullary nails, but additional studies are necessary to determine whether newer designs of cephalomedullary nails avoid the complications of older models, such as those used for periimplant fracture. In contrast to surgeon perception of technical ease, studies have shown there is a learning curve associated with cephalomedullary nails use [7, 27]. Parker et al. [27] found that the intramedullary nail had slightly increased operative and anesthesia time, increased radiographic screening time, and overall was more technically challenging with respect to placement of proximal and distal interlock screws. Their study calls into question the driving factors behind the changing practice pattern. Of the surgeons surveyed, nearly $1 / 3$ use only long nails. Despite a lack of evidence, the risk of periimplant fracture still drove decision-making for these surgeons (74\%). Short nail proponents chose ease of technique $(59 \%)$ and equivalency of outcomes to a long nail (24\%) as their reasons for using short cephalomedullary nails. Studies have suggested a higher risk of periimplant fracture with cephalomedullary nails compared with sliding hip screws [19, 25, 28], but Norris et al. [25] found that newer designs of cephalomedullary nails have a significantly decreased rate of periimplant fracture compared with their predecessors. Boone et al. [6] performed a retrospective study of intertrochanteric fractures treated with either a long or short cephalomedullary nail with minimum 1-year followup, finding no difference in the rate of periimplant fracture. With use of short cephalomedullary nails, there was significantly lower blood loss and rate of transfusion and shorter operative time. Further studies are necessary to determine whether short cephalomedullary nails have a higher incidence of periimplant fracture in long-term followup and whether this theoretical risk outweighs potential benefits of ease of use and lower perioperative morbidity compared with long cephalomedullary nails.

Although cost rarely was cited as an important decisionmaking factor by our cohort, it likely will become increasingly important as the economics of health care continue to evolve. A cephalomedullary nail costs approximately USD 900 to USD 1500 more than a sliding hip screw depending on region and institution [32]. Swart et al. [32] performed a cost-effectiveness analysis and found that sliding hip screws are likely more cost-effective than cephalomedullary nails for stable intertrochanteric fractures and those of questionable stability (AO Type 31A1 or A2), with fixation failure rate and implant cost being the most important factors in determining implant 
selection. Bohl et al. [5] found that patients treated with cephalomedullary nails had a shorter mean hospital stay compared with those treated with sliding hip screws (5 versus 7 days; $p<0.001$ ), introducing another factor that influences the relative cost-effectiveness of each implant.

Rather than cost, however, ease of use was the most common reason cited by respondents in our study for favoring cephalomedullary nails. If a surgeon is able to perform a cephalomedullary nail procedure with much greater ease and efficiency than a sliding hip screw procedure, this potentially could offset the cost of the implant. Chen et al. [7], in a study of Medicare enrollees from 1993 to 2007 , found that the outcome of cephalomedullary nailing has improved with time relative to sliding hip screws. This convergence in outcomes may be attributed to improved implant design and surgeon technique. Future studies may be directed toward observing whether the intraoperative parameters (such as operative time, blood loss, fluoroscopy time) change as surgeons become more proficient with use of cephalomedullary nailing. Egol et al. [9] proposed a treatment algorithm for intertrochanteric fractures, basing implant selection solely on AO classification, and found an $18 \%$ cost reduction per case without reduction in quality measures. Algorithms such as that of Egol et al. likely will become more important and widely used as greater emphasis is placed on healthcare cost containment.

We found that $2 / 3$ of surgeons allow immediate weightbearing after intertrochanteric fracture fixation, and surgeons who use cephalomedullary nails are more likely to allow immediate weightbearing. Although there are no high-quality prospective trials comparing weightbearing protocols, a couple of retrospective studies have shown that it is safe to allow early full weightbearing [16, 22]. Koval et al. [22] found a low rate of revision for loss of fixation (3\%) in a large cohort of elderly patients treated for stable or unstable intertrochanteric fractures using sliding hip screws. Herrera et al. [16] reviewed 551 intertrochanteric fractures in elderly patients who were allowed immediate weightbearing after surgery. They found that $1.4 \%$ of hips had screw cutout develop and $4 \%$ had collapsed into secondary varus at final followup. Early weightbearing and mobility may decrease postoperative morbidity and improve patient balance and independence without compromising fracture healing. Prospective and randomized studies are required to develop a definitive postoperative weightbearing protocol for patients with intertrochanteric fractures, and whether this has an effect on patient outcomes and length of hospital stay. Nearly all respondents $(99.5 \%)$ use postoperative chemical thromboprophylaxis in patients with intertrochanteric fractures. Although there are data on this topic for elective total joint arthroplasties, there are few studies regarding use of chemical thromboprophylaxis in patients with hip and intertrochanteric fractures [8, 10, 13, 29, 31]. Smith et al. [31] performed a prospective study in patients with operative hip and femur fractures who were treated with pharmacologic prophylaxis. They reported that $10 \%$ had deep vein thrombosis develop and increased risk was found with delay in surgery. The current American College of Chest Physicians guideline for hip fracture surgery recommends chemical thromboprophylaxis in addition to mechanical prophylaxis for a minimum of 10 to 14 days [10].

As with many new technologies, the early popularity of cephalomedullary nails was buoyed by promises of benefits $[15,24]$ that seemed to justify its attached price-tag. However, the case of the cephalomedullary nail is unique in that, while other innovations falter when the sheen of newness fades and they are revealed to be no better than what existed before them, the cephalomedullary nail has managed to overtake sliding hip screws. Our study showed that many orthopaedic surgeons believe cephalomedullary nails are a superior construct to sliding hip screws. To date, these beliefs do not have literature support and the orthopaedic community therefore has reached an impasse. We must decide whether the trend toward increased use of cephalomedullary nails should be allowed to continue in the absence of strong evidence or if we need to substantially change the way we currently approach intertrochanteric hip fractures. One key to this decision will be cost. As the population of the United States ages and intertrochanteric fractures become more common, it will become more important to use the most cost-effective surgical treatment for patients with hip fractures. Will it ultimately be more cost effective to support a two-implant model of treatment, where a treatment algorithm based on fracture pattern and other factors determine choice of implant, or will it be better to phase out sliding hip screws, and thus effectively increase the cost effectiveness of the cephalomedullary nail as surgeons become more proficient in their use and the cost of the implant depreciates as it becomes increasingly mainstream? If the decision is made to use a two-implant model, educational interventions will need to target not only academic centers and residents, but the community at large to ensure that all surgeons recognize the value of obtaining and maintaining proficiency with sliding hip screws and cephalomedullary nails. Future areas of study should focus on identifying whether the learning curve for cephalomedullary nailing has reached a plateau, further cost-benefit analysis of sliding hip screws versus cephalomedullary nails, and long versus short cephalomedullary nails, and creating a treatment algorithm or clinical practice guideline to optimize patient outcome without wasting healthcare resources. 


\section{References}

1. American Academy of Orthopaedic Surgeons. AAOS Orthopaedic Surgeon Census: Orthopaedic Practice in the U.S. 2014. Available at: http://www.aaos.org/research/orthocensus/census. asp. Accessed July 1, 2015.

2. Anglen JO, Weinstein JN; American Board of Orthopaedic Surgery Research Committee. Nail or plate fixation of intertrochanteric hip fractures: changing pattern of practice. A review of the American Board of Orthopaedic Surgery Database. J Bone Joint Surg Am. 2008;90:(Surgeons 2014).

3. Barton TM, Gleeson R, Topliss C, Greenwood R, Harries WJ, Chesser TJ. A comparison of the long gamma nail with the sliding hip screw for the treatment of AO/OTA 31-A2 fractures of the proximal part of the femur: a prospective randomized trial. $J$ Bone Joint Surg Am. 2010;92:792-798.

4. Bhandari M, Fong K, Sprague S, Williams D, Petrisor B. Variability in the definition and perceived causes of delayed unions and nonunions: a cross-sectional, multinational survey of orthopaedic surgeons. J Bone Joint Surg Am. 2012;94:e1091-1096.

5. Bohl DD, Basques BA, Golinvaux NS, Miller CP, Baumgaertner MR, Grauer JN. Extramedullary compared with intramedullary implants for intertrochanteric hip fractures: thirty-day outcomes of 4432 procedures from the ACS NSQIP database. J Bone Joint Surg Am. 2014;96:1871-1877.

6. Boone C, Carlberg KN, Koueiter DM, Baker KC, Sadowski J, Wiater PJ, Nowinski GP, Grant KD. Short versus long intramedullary nails for treatment of intertrochanteric femur fractures (OTA 31-A1 and A2). J Orthop Trauma. 2014;28:e96-e100.

7. Chen BS, Zhong W, Bhattacharyya T. Convergence of outcomes for hip fracture fixation by nails and plates. Clin Orthop Relat Res. 2013;471:1349-1355.

8. Della Valle CJ, Mirzabeigi E, Zuckerman JD, Koval KJ. Thromboembolic prophylaxis for patients with a fracture of the proximal femur. Am J Orthop (Belle Mead NJ). 2002;31:16-24.

9. Egol KA, Capriccioso CE, Konda SR, Tejwani NC, Liporace FA, Zuckerman JD, Davidovitch RI. Cost-effective trauma implant selection: AAOS exhibit selection. J Bone Joint Surg Am. 2014;96:e189.

10. Falck-Ytter Y, Francis CW, Johanson NA, Curley C, Dahl OE, Schulman S, Ortel TL, Pauker SG, Colwell CW Jr; American College of Chest Physicians. Prevention of VTE in orthopedic surgery patients: antithrombotic therapy and prevention of thrombosis, $9^{\text {th }}$ ed: American College of Chest Physicians Evidence-Based Clinical Practice Guidelines. Chest. 2012;141(2 suppl):e28S-e325S.

11. Forte ML, Virnig BA, Eberly LE, Swiontkowski MF, Feldman R, Bhandari M, Kane RL. Provider factors associated with intramedullary nail use for intertrochanteric hip fractures. J Bone Joint Surg Am. 2010;92:1105-1114.

12. Forte ML, Virnig BA, Kane RL, Durham S, Bhandari M, Feldman R, Swiontkowski MF. Geographic variation in device use for intertrochanteric hip fractures. J Bone Joint Surg Am. 2008;90: 691-699.

13. Girasole GJ, Cuomo F, Denton JR, O'Connor D, Ernst A. Diagnosis of deep vein thrombosis in elderly hip-fracture patients by using the duplex scanning technique. Orthop Rev. 1994;23:411-416.

14. Gross M. Polling surgeons. Can J Surg. 2005;48:433.

15. Halder SC. The Gamma nail for peritrochanteric fractures. J Bone Joint Surg Br. 1992;74:340-344.

16. Herrera A, Domingo J, Martinez A. Results of osteosynthesis with the ITST nail in fractures of the trochanteric region of the femur. Int Orthop. 2008;32:767-772.

17. Hung WW, Morrison RS. Hip fracture: a complex illness among complex patients. Ann Intern Med. 2011;155:267-268.
18. Jones HW, Johnston P, Parker M. Are short femoral nails superior to the sliding hip screw? A meta-analysis of 24 studies involving 3,279 fractures. Int Orthop. 2006;30:69-78.

19. Kaplan K, Miyamoto R, Levine BR, Egol KA, Zuckerman JD. Surgical management of hip fractures: an evidence-based review of the literature. II: Intertrochanteric fractures. J Am Acad Orthop Surg. 2008;16:665-673.

20. Knobe M, Gradl G, Ladenburger A, Tarkin IS, Pape HC. Unstable intertrochanteric femur fractures: is there a consensus on definition and treatment in Germany? Clin Orthop Relat Res. 2013;471:2831-2840.

21. Kokoroghiannis C, Aktselis I, Deligeorgis A, Fragkomichalos E, Papadimas D, Pappadas I. Evolving concepts of stability and intramedullary fixation of intertrochanteric fractures: a review. Injury. 2012;43:686-693.

22. Koval KJ, Friend KD, Aharonoff GB, Zukerman JD. Weight bearing after hip fracture: a prospective series of 596 geriatric hip fracture patients. J Orthop Trauma. 1996;10:526-530.

23. Kregor PJ, Obremskey WT, Kreder HJ, Swiontkowski MF. Unstable pertrochanterirc femoral fractures. J Orthop Trauma. 2014;28(suppl 8):S25-28.

24. Lindsey RW, Teal P, Probe RA, Rhoads D, Davenport S, Schauder K. Early experience with the gamma interlocking nail for peritrochanteric fractures of the proximal femur. J Trauma. 1991;31:1649-1658.

25. Norris R, Bhattacharjee D, Parker MJ. Occurrence of secondary fracture around intramedullary nails used for trochanteric hip fractures: a systematic review of 13,568 patients. Injury. 2012;43:706-711.

26. Palm H, Jacobsen S, Sonne-Holm S, Gebuhr P; Hip Fracture Study Group. Integrity of the lateral femoral wall in intertrochanteric hip fractures: an important predictor of a reoperation. J Bone Joint Surg Am. 2007;89:470-475.

27. Parker MJ, Bowers TR, Pryor GA. Sliding hip screw versus the Targon PF nail in the treatment of trochanteric fractures of the hip: a randomised trial of 600 fractures. J Bone Joint Surg Br. 2012;94:391-397.

28. Parker MJ, Handoll HH. Gamma and other cephalocondylic intramedullary nails versus extramedullary implants for extracapsular hip fractures in adults. Cochrane Database Syst Rev. 2010;9:CD000093.

29. Roberts TS, Nelson CL, Barnes CL, Ferris EJ, Holder JC, Boone DW. The preoperative prevalence and postoperative incidence of thromboembolism in patients with hip fractures treated with dextran prophylaxis. Clin Orthop Relat Res. 1990;255;198-203.

30. Saudan M, Lubbeke A, Sadowski C, Riand N, Stern R, Hoffmeyer P. Pertrochanteric fractures: is there an advantage to an intramedullary nail? A randomized, prospective study of 206 patients comparing the dynamic hip screw and proximal femoral nail. J Orthop Trauma. 2002;16:386-393.

31. Smith EB, Parvizi J, Purtill JJ. Delayed surgery for patients with femur and hip fractures: risk of deep venous thrombosis. $J$ Trauma. 2011;70:E113-116.

32. Swart E, Makhni EC, Macaulay W, Rosenwasser MP, Bozic KJ. Cost-effectiveness analysis of fixation options for intertrochanteric hip fractures. J Bone Joint Surg Am. 2014;96:1612-1620.

33. Tawari AA, Kempegowda H, Suk M, Horwitz DS. What makes an intertrochanteric fracture unstable in 2015? Does the lateral wall play a role in the decision matrix? J Orthop Trauma. 2015;29(suppl 4): S4-9.

34. Utrilla AL, Reig JS, Munoz FM, Tufanisco CB. Trochanteric gamma nail and compression hip screw for trochanteric fractures: a randomized, prospective, comparative study in 210 elderly patients with a new design of the gamma nail. J Orthop Trauma. 2005;19:229-233. 